\title{
A rare case of invasive cystic hypersecretory carcinoma of the breast post neoadjuvant therapy: Case report and literature review
}

Ghada Mohamed ${ }^{1,2^{*}}$, Aya ElMahs ${ }^{2,3}$ and Haddel Ahmed ${ }^{2}$

*Correspondence: dr.ghada.elshafaee@gmail.com

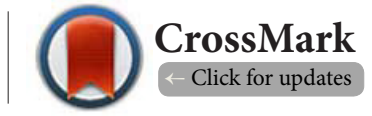

'Department of Pathology, National Cancer Institute, Cairo University, Egypt.

${ }^{2}$ Department of Pathology, Baheya Center For Early Detection and Treatment of Breast Cancer, Egypt.

${ }^{3}$ Department of Pathology, Helwan Faculty of Medicine, Helwan University, Egypt.

\begin{abstract}
Invasive cystic hypersecretory carcinoma (invasive $\mathrm{CHC}$ ) of the breast is extremely rare and underdiagnosed type of breast cancer that is not included in the WHO classification of breast tumors. It has distinct gross and microscopic features. To the best of our knowledge, only 20 cases have been reported in the English literatures. So far, no data have been documented about its response to chemotherapy.

Herein we reported another new case, 65-year-old, of invasive $\mathrm{CHC}$ received neoadjuvant chemotherapy followed by mastectomy with poor therapy response. On microscopic examination, our case showed the Coexistences of the three forms of cystic hypersecretory lesions together [Cystic hypersecretory hyperplasia, $\mathrm{CHC}$ and invasive $\mathrm{CHC}$ ]. Axillary lymph node metastasis is rarely reported and only five cases presented by axillary lymph node metastasis in the literatures. All were stage N1. Our case is also unique in that it was presented with stage N3 lymph node metastasis, that involved both axillary and infraclavicular lymph nodes. In conclusion, invasive $\mathrm{CHC}$ is a rare type of breast cancer and pathologist should be aware of it to help in the differential diagnosis, especially on examining a biopsy material.
\end{abstract}

Keywords: Cystic hypersecretory carcinoma, breast cancer, neoadjuvant therapy

\section{Background}

Invasive cystic hypersecretory carcinoma (Invasive $\mathrm{CHC}$ ) is a rare subtype of breast duct carcinoma that is not included in the recent WHO classification of tumors of the breast, 2018. It is one of the spectra of breast cystic hypersecretory lesions, which also includes cystic hypersecretory hyperplasia $(\mathrm{CHH})$, cystic hypersecretory hyperplasia with atypia and cystic hypersecretory carcinoma insitu (CHC) [1]. The insitu carcinoma type usually behave in an indolent manner, but can give rise to invasive carcinoma, where it is usually of high grade [2]. These lesions have a characteristic gross finding of multiple cysts filled with gelatinous material. On microscopic examination, they all show cystically dilated ducts filled with homogenous eosinophilic material often with peripheral retraction halo, resembling thyroid follicles. Presence of solid areas suggests invasive component
[1]. So far in the English literatures, only 20 cases of invasive $\mathrm{CHC}$ have been reported [3]. No report till now documented the response of invasive $\mathrm{CHC}$ to chemotherapy. Moreover, its biologic behavior and prognosis are still unknown due to the few numbers of the reported cases. Herein we reported another case of invasive $\mathrm{CHC}$ received neoadjuvant therapy followed by modified radical mastectomy (MRM).

\section{Case presentation}

Our case is a female patient, 65-year-old, diabetic and hypertensive with positive family history of breast cancer. She had a long history of neglected breast small mass for about 4 years prior to seeking any medical advice. She was admitted to our hospital: Baheya Center, Egypt and presented with a left breast large mass with multiple skin nodules and ulceration.

(C) 2021 Mohamed et al; licensee Herbert Publications Ltd. This is an Open Access article distributed under the terms of Creative Commons Attribution License (http://creativecommons.org/licenses/by/3.0). This permits unrestricted use, distribution, and reproduction in any medium, provided the original work is properly cited. 
Nipple and areola complex were infiltrated. Mammographic and ultrasonographic exams showed a locally advanced malignant-looking mass $58 \times 30 \times 28 \mathrm{~mm}$ with skin thickening, together with pathologic axillary and infraclavicular lymph nodes [stage N3]. However, no other metastatic lesions were found by bone scan, abdominal and chest $\mathrm{CT}$ examinations [stage M0].

Biopsy was taken and the initial diagnosis was invasive breast carcinoma of NST, grade III. Its hormonal profile showed a triple negative carcinoma (ER -, PR - and HER2/neu -).

The patient then received full course of chemotherapy, Epirubicin and Cyclophosphamide(EC), followed by Paclitaxel chemotherapy, however the tumor showed progression on treatment. After that, a modified radical mastectomy was done.

\section{Pathological findings}

Gross examination revealed Lt. breast with multiple skin nodules and skin ulceration with infiltrated nipple (Figure 1). Serial sectioning revealed a firm solid and white tumor $(120 \times 70 \times 20 \mathrm{~mm})$, in the superficial part of the breast infiltrating the skin with scattered cystic areas. Resected deep margin

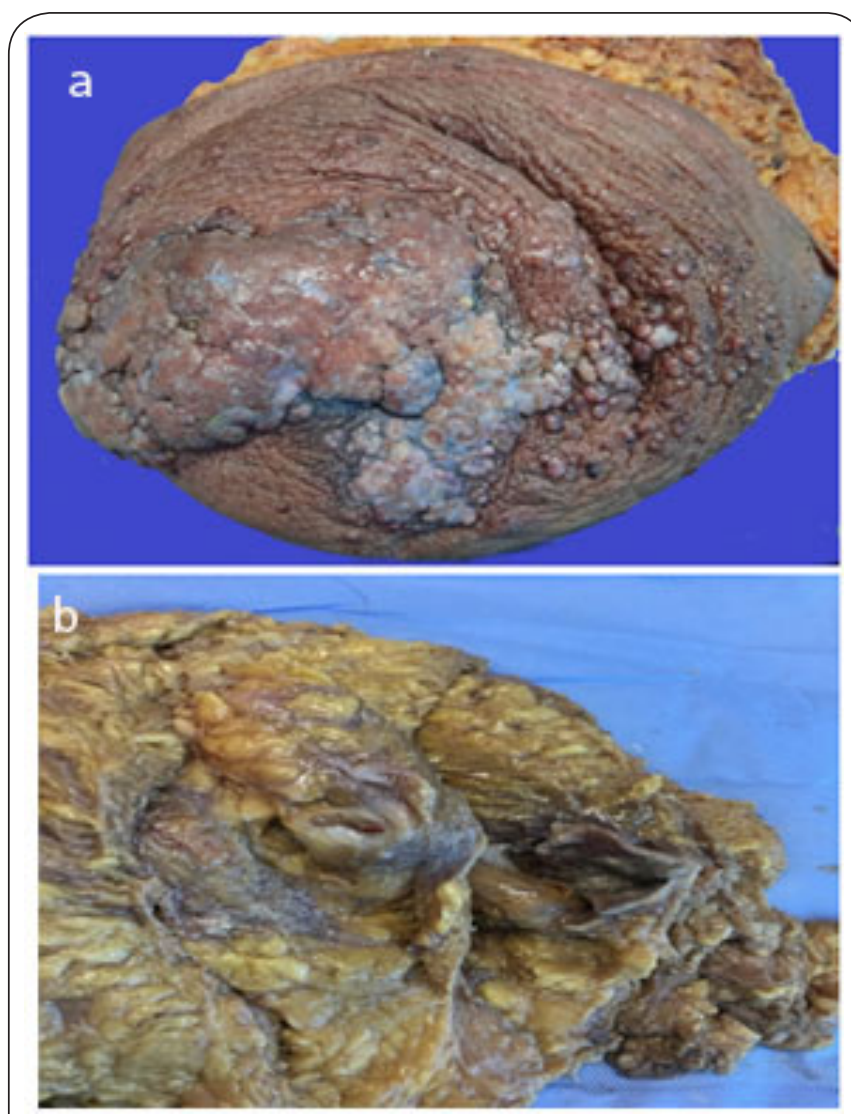

Figure 1. Gross examination of the excised specimen after neoadjuvant chemotherapy.

a) There are multiple skin nodules with tumor ulceration and nipple infiltration.

b) Few tumor cyts in the breast tissue. was $1 \mathrm{~cm}$. The largest dissected and infiltrated axillary lymph node was $30 \times 25 \mathrm{~mm}$.

Microscopic examination from the skin nodules showed multiple dilated cysts filled with colloid-like eosinophilic material with occasion peripheral scalloping (Figure 2a-2c). Rest of breast tissue showed multiple nodules of cystically dilated ducts containing eosinophilic hyaline secretions together with microcystic and solid invasive patterns. Lining of the cystic structures showed papillary and micropapillary patterns with moderate nuclear atypia (Figure $\mathbf{2} \mathbf{d}$ and $\mathbf{2 e}$ ). There was an associated $\mathrm{CHH}$ component, where cysts are lined by flat epithelium with eosinophilic cytoplasm and round to oval vesicular benign-looking nuclei (Figure $\mathbf{2 h}$ ). Invasive tumor areas showed microcystic and solid growth pattern with lymphovascular invasion. Tumor cells exhibited moderate nuclear anaplasia. Tumor necrosis was seen with areas of therapy effect including stromal fibrohyalinosis and chronic inflammation. Ductal carcinoma insitu component predominated in the form of papillary and micropapillary types, however focal cribriform pattern was also seen (Figure 2g). Nipple was infiltrated by tumor groups, but no Paget cells. Skin and deep margins were free from tumor infiltration. Overall residual cellularity was $90 \%$ with necrotic areas denoting chemotherapy effect (Figure 2f). Axillary I. nodes showed metastatic deposit in $(13 / 17$, stage pN3) with the same cystic morphology as in the primary tumor (Figure 2i). There was capsular infiltration and extension to the perinodal fat. No evidence of chemotherapy effect in the axillary lymph node. Residual cancer burden was calculated and revealed class-III (extensive residual burden).

Immunohistochemical study revealed positive reaction of the tumor cells to $\mathrm{S100}$. Scattered tumor areas showed positive reaction to CK5/6. KI-67 LI was (40\%) (Figure 3).

\section{Discussion}

Cystic hypersecretory lesions of the breast ranged from clearly benign disease $(\mathrm{CHH}), \mathrm{CHH}$ with atypia, insitu carcinoma $(\mathrm{CHC})$ and the invasive type. $\mathrm{CHC}$ is differentiated from $\mathrm{CHH}$ by the presence of atypical epithelial cells lined the cysts with micropapillary pattern, while $\mathrm{CHH}$ is lined by a flat or columnar epithelium without nuclear atypia. Invasive $\mathrm{CHC}$ tends to be of high grade. Most of the reported cases were of the insitu carcinoma type, which has an indolent course. $\mathrm{CHC}$ with microinvasive carcinoma was reported in three cases $[2,4]$. Invasion is known by the presence of solid nests $[\mathbf{1 , 5}]$.

So far, to the best of our knowledge, only 20 cases of invasive $\mathrm{CHC}$ were reported. Ages of the patients ranged 32-70 year [3]. Five of these cases showed axillary lymph node macrometastasis, and all were stage $\mathrm{N} 1$. Our case is the $21^{\text {st }}$ case of the reported invasive $\mathrm{CHC}$, the sixth case with axillary lymph node macrometastasis and the first case to present with stage N3 nodal metastasis [with involvement of both axillary and infraclavicular lymph nodes].

None of the reported cases examined the response of this type of carcinoma to the neoadjuvant chemotherapy. One of 


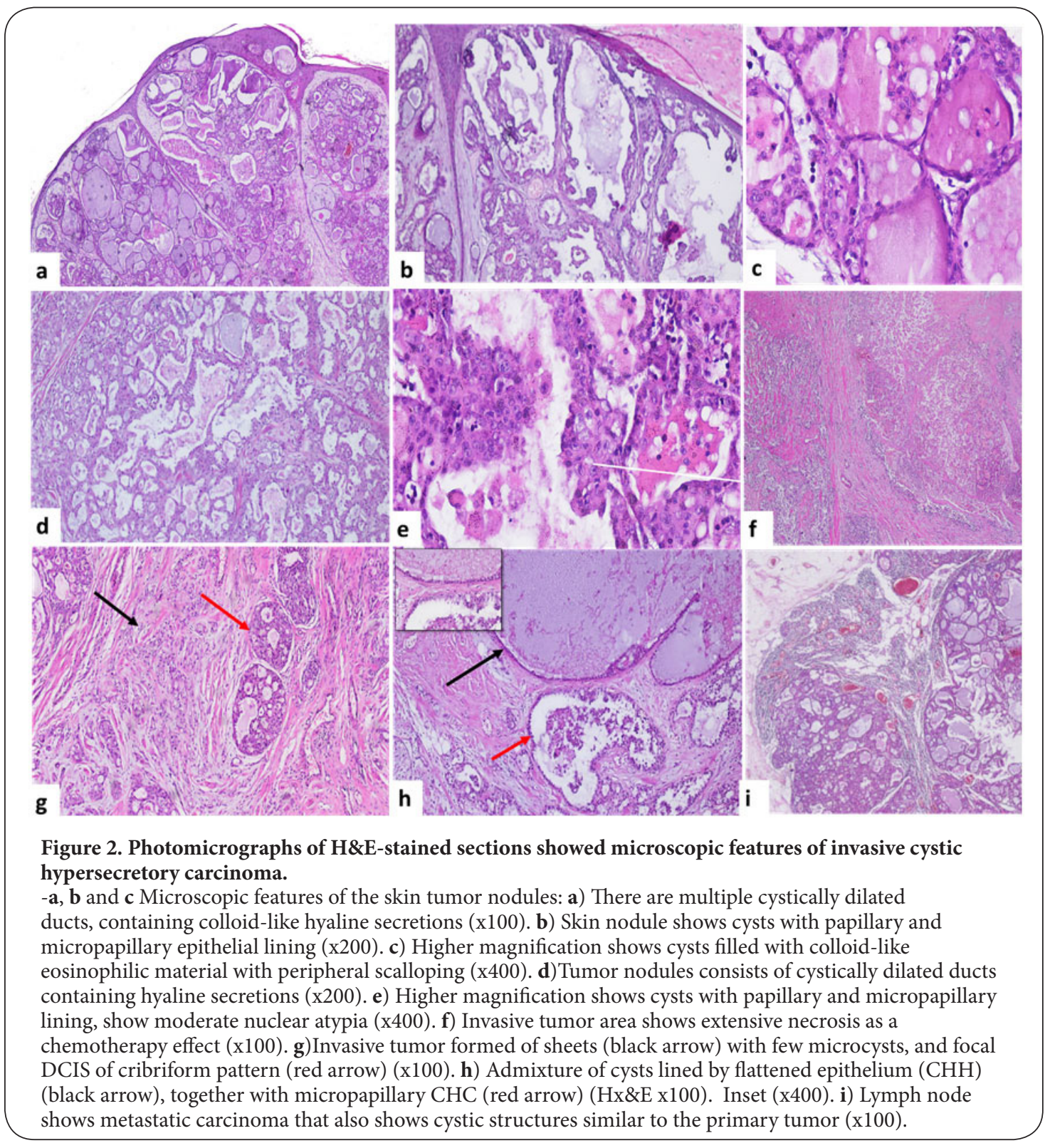

the reported cases presented by a locally advanced tumor with bone metastasis, then received chemo and radiotherapy, however died 9 months later [1]. In the present report, we assessed the chemotherapy response after receiving an aggressive course of neoadjuvant chemotherapy followed by mastectomy. Clinically, our case showed a progressive course on chemotherapy. Pathologically, it revealed extensive residual cancer burden [RCB class III] with no therapy effect in the axillary lymph node metastasis.

The current case presented by a locally advanced cancer with skin nodules and ulceration [stage pT4]. Skin ulceration was also one of the initial presentations in one report [3].

Interestingly, microscopic examination of our case documented the admixture of the three forms of cystic hypersecre- tory lesions together $[\mathrm{CHH}, \mathrm{CHC}$ and invasive $\mathrm{CHC}$. This was also reported in the literature [6], thus it may suggest disease progression from $\mathrm{CHH}$ to invasive carcinoma. However, no cases till now documented a progression from $\mathrm{CHH}$ disease to $\mathrm{CHC}$.

It is known that $\mathrm{CHC}$ has an indolent course. Guerry et al., reported that on follow up of 29 cases of $\mathrm{CHC}, 19$ of them live free without recurrence after mastectomy, with a mean follow up 8 years [1]. This could explain the long history of our case with a neglected breast mass for up to 4 years prior to present with skin ulceration and metastasis in the I. nodes. Hormonal profile of our case showed triple negative carcinoma (ER -, PR-, HER2/neu -). Limited data is available in the literatures about the hormonal profile of this invasive carcinoma, but similar to us, Sahoo et al., reported a triple-negative profile [7]. 


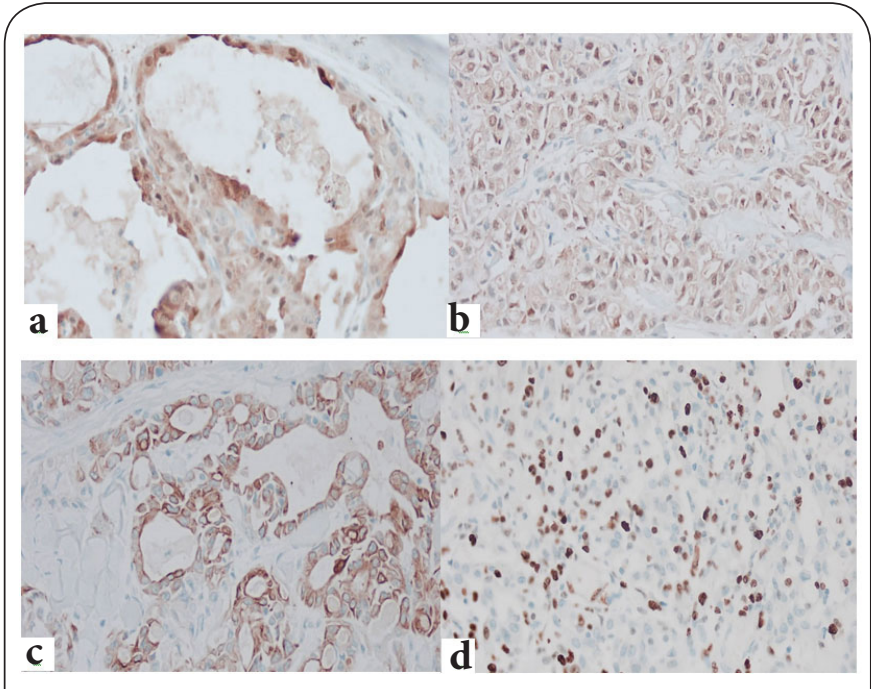

Figure 3. Photomicrographs of immunostained sections (x400).

$\mathrm{S} 100$ is positive in both the cystic component of the tumor (a) and in the invasive tumor (b). (c) In some areas, tumor showed positive reaction to CK5/6. (d) KI-67 LI in the invasive tumor area.

On the other hand, Young Zhong et al., reported (ER +, PR-, HER2/ neu -) [8], while Sun et al., showed ER-, PR- and HER2 positive [3]. Similar to other reported cases [8,9], our case showed positive reaction of the tumor cells to $\mathrm{S} 100$, and scattered areas were positive to CK5/6. This profile is similar to secretory carcinoma. However, it can be differentiated from invasive $\mathrm{CHC}$ by the presence of a predominant microcystic/honeycomb invasive pattern. Additionally, its insitu carcinoma component is typically of cribriform and solid pattern, unlike $\mathrm{CHC}$ which typically shows predominant large cystically dilated structures. Other differential diagnosis includes mucocele-like lesion, apocrine carcinoma, apocrine DCIS, and mucinous carcinoma. However, the described distinct gross and microscopic pictures help in the differential diagnosis. It could be difficult to differentiate this tumor from its mimics on core biopsy, thus being aware of this tumor can help in biopsy-based initial diagnosis. Excision is usually required for confirmation.

\section{Conclusion}

Invasive $\mathrm{CHC}$ is a rare and underdiagnosed breast tumor that has distinct gross and microscopic findings. Pathologist should be aware of it to help in the differential diagnosis, especially on examining a biopsy material. Most of the reported cases showed almost triple negative morphology Invasive CHC is an aggressive type of breast cancer, and its response to chemotherapy seems to be poor as we reported. While CHC is treated by excision, until now there is no specific chemotherapy regimen to the invasive $\mathrm{CHC}$.

\section{List of abbreviations}

CHC: Cystic hypersecretory carcinoma
WHO: World health organization

$\mathrm{CHH}$ : Cystic hypersecretory hyperplasia

MRM: Modified radical mastectomy

CT: Computed tomography

NST: No special type.

ER: Estrogen receptor

PR: Progesterone receptor

HER2/neu: Human epidermal growth factor receptor 2

EC: Epirubicin and cyclophosphamide

RCB: Residual cancer burden

\section{Competing interests}

The authors declare that they have no competing interests.

\section{Authors' contributions}

\begin{tabular}{|l|c|c|c|}
\hline Authors' contributions & GM & AE & HA \\
\hline Research concept and design & $\sqrt{ }$ & -- & -- \\
\hline Collection and/or assembly of data & $\sqrt{ }$ & -- & $\sqrt{ }$ \\
\hline Data analysis and interpretation & $\sqrt{ }$ & $\sqrt{ }$ & -- \\
\hline Writing the article & $\sqrt{ }$ & -- & $\sqrt{ }$ \\
\hline Critical revision of the article & $\sqrt{ }$ & $\sqrt{ }$ & $\sqrt{ }$ \\
\hline Final approval of article & $\sqrt{ }$ & $\sqrt{ }$ & $\sqrt{ }$ \\
\hline Statistical analysis & -- & -- & -- \\
\hline
\end{tabular}

\section{Publication history}

Editor: Khush Mittal, New York University School of Medicine, USA. Received: 08-May-2021 Final Revised: 28-June-2021

Accepted: 01- July-2021 Published: 21-July-2021

\section{References}

1. Guerry P, Erlandson RA, Rosen PP.Cystic hypersecretory hyperplasia and cystic hypersecretory duct carcinoma of the breast. Cancer. 1988; 61:1611- 20.

2. D’Alfonso TM, Ginter PS, Liu YF, Shin SJ.Cystic hypersecretory (in situ) carcinoma of the breast: a clinicopathologic and immunohistochemical characterization of $\mathbf{1 0}$ cases with clinical follow-up. Am J Surg Pathol. 2014;38(1):45-53.

3. Sun J, Wang X \& Wang, C.Invasive cystic hypersecretory carcinoma of the breast: a rare variant of breast cancer: a case report and review of the literature. BMC Cancer.2019; 19, 31.

4. Chen DB, Kan X.Cystic hypersecretory carcinoma with microinvasive carcinoma and cystic hypersecretory hyperplasia of breast: report of a case. Zhonghua Bing Li Xue Za Zhi. Chinese. 2010;39(1):54 -5. 14.

5. Rosen PP, Scott M.Cystic hypersecretory duct carcinoma of the breast. Am J Surg Pathol. 1984;8(1):31-41.

6. Lee JS, Lee YJ.Invasive cystic hypersecretory carcinoma of the breast: a case report. J Korean Med Sci. 2004;19(1):149-51.

7. Sahoo N, Mishra P, Patra S, Sasmal PK.Invasive Cystic Hypersecretory Carcinoma of Breast: A Rare and Under Diagnosed Variant of Ductal Carcinoma.J Clin Diagn Res. 2017;11(6):ED16-ED17.

8. Yongzhong $Y$, Xianbiao $S$, Yixin, $Z$, Xin $Y$, Jingmei $W$, Huiping $Y$ and Shouju W.Invasive Cystic Hypersecretory Carcinoma of Breast. Biomed J Sci \& 
Mohamed et al., Journal of Histology \& Histopathology 2021,

Tech Res. 2018;2(2)2477-2480.

9. Bi R, Cheng Y, Yu B, Shui R, Yang W, Xu X.Clinicopathologic features of cystic hypersecretory lesion of the breast.Zhonghua Bing Li Xue Za Zhi. Chinese.2014;43(1):25-9.

\section{Citation:}

Mohamed G, ElMahs A and Ahmed H. A rare case of invasive cystic hypersecretory carcinoma of the breast post neoadjuvant therapy: Case report and literature review. J Histol Histopathol. 2021; 8:2.

http://dx.doi.org/10.7243/2055-091X-8-2 